motor cortical outputs to the right and left first dorsal interosseous (FDI) and right abductor digiti minimi (ADM) muscles of the reading hand in 6 blind proficient Braille readers studied at the National Institute of Neurological Disorders and Stroke, NIH, Bethesda, MD. All subjects had learned Braille before age 13, using the right index finger for character recognition and the left index for line keeping. Comparison of cortical output maps obtained on working days and off days showed that the maps for the FDI of the reading hand were significantly larger in the evening after the working shift than in the morning after having not worked for 2 days. The map shrunk following vacation days and enlarged following a return to work. On control days, the motor threshold for the muscles stayed constant, whereas on the work day, the motor thresholds for the right FDI were significantly lower in the evening study session after practice than in the morning test before practice. The results illustrate the rapid modulation in motor cortical outputs effected by Braille reading. (Pascual-Leone $\mathrm{A}$, Hallett $\mathrm{M}$ et al. The role of reading activity on the modulation of motor cortical outputs to the reading hand in Braille readers. Ann Neurol December 1995;38:910-915). (Respond: Dr Pascual-Leone, Unidad de Neurobiologia, Departemente Fisiologia, Universidad Valencia, Avda Blasco Ibanez 17, Valencia 46010, Spain: or Dr Hallett, NINDS, NIH, Bldg 10, Rm 5N226, 10 Center DR MSC 1428, Bethesda, MD 20892).

COMMENT. The authors emphasize the critical importance of timing when looking for changes in neural networks associated with learning skills. Learning a new skill requires plastic changes and rapid modulation of intracortical connections that result in temporary enlargement of the cortical motor output. These neurophysiological changes are supported by PET scanning studies in the same laboratory, showing that Braille reading is associated with an increased activation of the sensorimotor and striate cortex contralateral to the reading hand. (Sadato $\mathrm{N}$ et al. 1995).

\title{
INTELLIGENCE AND MRI CHANGES IN NEUROFIBROMATOSIS
}

Brain MRI changes were studied in relation to intelligence in a group of 28 children, aged 4 to 16 years, with neurofibromatosis 1 . The mean FS IQ on the Wechsler scales (WPPSI, WISC-R) was 89 (range 54-148), mean VS was 95 and PS 84. Eight children who had neurological disease (epilepsy, hydrocephalus, tumor, or post-irradiation) scored significantly lower on IQ tests than the 20 without neurological disease. Eighteen children with hyperintense T2 weighted foci had a mean FS IQ comparable with the 10 without spots. In the group without neurologic disease, the 10 showing hyperintense spots had a higher mean FS IQ than 10 without. There was no significant relation between the number or location of T2 weighted foci and FS IQ (Legius E et al. Neurofibromatosis type 1 in childhood: correlation of MRI findings with intelligence. I Neurol Neurosurg Psychiatry December 1995;59:635-640). (Respond: Dr Eric Legius, Center for Human Genetics, University Hospital Gasthuisberg, Herestraat 49, 3000 Leuven, Belgium).

COMMENT. Previous studies have shown that the mean full scale IQ in children with neurofibromatosis 1 is shifted to the left, between 88 and 94 . T2 weighted hyperintense foci in the brain MRI were not correlated with intelligence of children with neurofibromatosis 1 in the present study, and similar results have been reported from other centers. (see Progress in Pediatric Neurology I, PNB Publishers, 1991, pp376-77). However, the authors cite two recent reports showing a significant correlation between a lowered IQ 
and the presence of T2 weighted hyperintensities in the MRI of 52 affected children who had no major neurologic complication or frank retardation. Further studies are needed to define the significance of these hyperintense foci.

\section{SEIZURE DISORDERS}

\section{CSF GLUCOSE IN FEBRILE CONVULSIONS}

The effects of convulsion and fever on the CSF and blood glucose concentrations in febrile and non-febrile children, with and without convulsions, have been studied at the Department of Paediatrics, Kuopio University Hospital and Department of Pharmacology and Toxicology, University of Kuopia, Kuopio, Finland. The concentration of glucose in the CSF was significantly higher in febrile children with and without convulsions than in non-febrile, non-convulsive children. Both fever and convulsions increased the CSF glucose levels. The body temperature plotted against the CSF glucose showed a linear correlation. Blood glucose parallelled CSF levels in all groups. Hyperglycemia and elevated CSF glucose in febrile convulsions are apparently secondary to both the fever and convulsion, not the convulsion alone. (Kiviranta $\mathrm{T}$ et al. The role of fever on cerebrospinal fluid glucose concentration of children with and without convulsions. Acta Paediatr 1995;84:1276-9). (Respond: Dr T Kiviranta, Department of Paediatrics, Kuopio University Hospital, PO Box 1777, FIN-70211, Kuopio, Finland).

COMMENT. Of 110 patients with febrile seizures examined personally, the cerebrospinal fluid was essentially normal in 86 tested. The concentration of sugar was greater than $80 \mathrm{mg} / 100 \mathrm{ml}$ in 24 patients and $100 \mathrm{mg} / 100 \mathrm{ml}$ or higher in 11. (Millichap JG et al. 1960). A review of the literature in the $1960 \mathrm{~s}$ revealed 18 publications between 1934 and 1964, which included the CSF findings of 500 children with febrile convulsions. Elevations of CSF sugar were found in only three reports, in addition to my own study, the first in 1938, and these involved 37 of 68 patients tested. (Millichap JG. Febrile Convulsions, New York, Macmillan, 1968). The present study attempts to elucidate the mechanism of the increased CSF sugar concentration found in some children with febrile convulsions. Both fever and convulsion were found to have a role in elevating the CSF sugar levels.

\section{PAINFUL HAND SEIZURES}

A 14-year-old boy with habitual painful seizures of the backs of both hands since age 4 is reported from the Department of Pediatric Neurology, Osaka Medical Center, Japan. He had three febrile convulsions from one to three years of age. Painful hand seizures occurred 5 - 15 times daily, lasting 15 - 60 seconds, and occasionally followed by loss of consciousness and postictal confusion but no secondarily generalized seizures. Seizures were resistant to conventional medications until 13 years of age, when they showed some response to polytherapy with carbamazepine, valproate, and clorazepate. Interictal EEG showed frequent spikes and spike-waves over the right frontopolar area with spread to the left frontal region. Ictal EEG showed right temporal $4-6 \mathrm{~Hz}$ rhythmic activity after a pain sensation. CT and MRI were normal. SPECT showed right temporal hypoperfusion. These secondary sensory seizures were thought to originate from the S2A area. (Otani K et al. Bilateral painful epileptic seizures of the hands. Dev Med Child Neurol Oct 1995;37:933- 